\title{
Obesity-Induced Structural and Neuronal Plasticity in the Lateral Orbitofrontal Cortex
}

\author{
Jennifer L Thompson ${ }^{1,2}$, Michael Drysdale², Corey Baimel ${ }^{1,2}$, Manpreet Kaur², Taigan MacGowan², \\ Kimberley A Pitman ${ }^{1,2}$ and Stephanie L Borgland*,2 \\ 'Department of Anesthesiology, Pharmacology and Therapeutics, University of British Columbia, Vancouver, British Columbia, Canada; ${ }^{2}$ Hotchkiss \\ Brain Institute, University of Calgary, Calgary, Alberta, Canada
}

\begin{abstract}
The orbitofrontal cortex (OFC) integrates sensory information with the current value of foods and updates actions based on this information. Obese humans and rats fed a cafeteria diet have impaired devaluation of food rewards, implicating a potential obesity-induced dysfunction of the OFC. We hypothesized that obesity alters OFC pyramidal neuronal structure and function and reduces conditioned suppression of feeding. Rats were given restricted (I h/day), extended ( $23 \mathrm{~h} /$ day) or no (chow only) access to a cafeteria diet and tested for a conditioned suppression of feeding. Golgi-cox impregnation and whole-cell patch clamp experiments were performed in lateral OFC pyramidal neurons of rats from the 3 feeding groups. Rats with 40 days of extended, but not restricted, access to a cafeteria diet became obese and continued to feed during foot shock-predicting cues. Access to a cafeteria diet induced morphological changes in basilar dendrites of lateral OFC pyramidal neurons. While there were no alterations in excitatory synaptic transmission underlying altered spine density, we observed a more depolarized resting membrane potential. This was accompanied by decreased inhibitory synaptic transmission onto lateral OFC pyramidal neurons due to decreased release probability at GABAergic inputs. These changes could underlie the inability of the OFC to encode changes in the motivation value of food that is observed in obese rodents and humans. Neuropsychopharmacology (2017) 42, 1480-1490; doi:I0.1038/npp.2016.284; published online I 8 January 2017
\end{abstract}

\section{INTRODUCTION}

Worldwide obesity has doubled since the 1980s (Finucane et al, 2011). While there are several causes for this, a predominant hypothesis is that increased availability of low cost, easily accessible, highly palatable foods drives overeating. Overeating is the largest determinant of obesity (Swinburn et al, 2009), yet we understand very little of the neural mechanisms underlying why individuals continue to consume food regardless of satiety. The motivational value of food scales with hunger state. For example, the incentive value of food is lower during satiety, but enhanced when one is hungry or exposed to a variety of palatable food options (Berridge, 1991; Rolls et al, 1988; Stoeckel et al, 2007). In contrast, obese human subjects are not sensitive to the changes in motivational value of food and continue to eat in a habit-like fashion even if food has been devalued with satiety (Horstmann et al, 2015). Similarly, rodents on a cafeteria diet continue to consume food when the motivational value of food has been devalued with satiety (Reichelt et al, 2014) or by the presence of aversive cues (Johnson and Kenny, 2010).

* Correspondence: Dr SL Borgland, Hotchkiss Brain Institute, University of Calgary, 3330 Hospital Dr NW Calgary, Alberta T2N 4NI, Canada, Tel: + | 403220 6967, Fax: +4032832700, E-mail: s.borgland@ucalgary.ca Received 30 September 20 16; revised 22 November 20 I6; accepted I 8 December 2016; accepted article preview online 2 January 2017
The orbitofrontal cortex (OFC) is implicated in reward valuation and decision-making. OFC neurons hold valuebased information 'online' to guide future decision- making and thus estimate the likelihood of specific outcomes to guide future responses (Wilson et al, 2014). Given that the OFC receives significant sensory information and contains neurons that respond to taste and olfaction (Rolls, 2012), the OFC likely integrates signals about the sensory properties of food (taste, texture, smell) with expected outcomes. OFC lesions or inactivation are associated with impairment in either satiety-induced (Gremel and Costa, 2013; Rudebeck et al, 2013) or $\mathrm{LiCl}$ (sickness)-induced food devaluation (Schoenbaum et al, 2003). Thus, the OFC registers the current value of foods and updates actions based on this information. Because obese humans and rats fed a cafeteria diet have impaired devaluation of food rewards, dysfunction in the OFC may be implicated as a potential driver of overeating.

To examine the effects of obesity on OFC function, we used an obesogenic model previously described in (Johnson and Kenny, 2010). Three groups were given differential access $(0,1$, or $24 \mathrm{~h})$ to a 'cafeteria style' diet consisting of palatable energy-dense food readily available for human consumption in addition to ad libitum access to chow for 40d. The restricted $1 \mathrm{~h}$ access provides an important comparison as one can dissociate effects due to obesity, as previous reports have indicated that restricted access to a cafeteria diet is not obesogenic (Johnson and Kenny, 2010). 
We hypothesized that diet-induced obesity due to extended access to a cafeteria diet alters the structure and function of OFC pyramidal neurons.

\section{MATERIALS AND METHODS}

\section{Subjects}

Male Long-Evans rats (Charles River Laboratories, Quebec, Canada) were obtained at approximately postnatal day 60 (285-325 g at the start of all experiments). Rats were housed individually on a 12:12 reverse light dark cycle (lights on at 8:00 am). Rats were permitted ad libitum access to standard chow (5P14 LabDiet, St Louis, MO) and water for the duration of the experiment. All experimental procedures were approved by the Animal Care Committee of the University of Calgary under the guidelines of the Canadian Council on Animal Care.

\section{Cafeteria Diet}

All rats were housed individually on paper bedding (Alphapads, Lawrenceville, GA) to prevent foods from being soiled with loose bedding materials. The cafeteria diet consisted of Kirkland beef hotdogs, Froot Loops, Timbit donut holes, Kraft peanut butter, and Doritos. All food was weighed on completion of the feeding session and adjusted for dehydration. Caloric intake from the various macronutrients was calculated using the nutritional information provided by the manufacturer (Supplementary Table 1). Rats were exposed to the cafeteria diet for $1 \mathrm{~h}$ per day (restricted access), $23 \mathrm{~h}$ per day (extended access) or $0 \mathrm{~h}$ per day (chow only) for a minimum of 40 days. Food was replenished daily $2 \mathrm{~h}$ into their dark cycle. The $1 \mathrm{~h}$ exposure for restricted access rats was presented between $2-3 \mathrm{~h}$ into their dark cycle. Because each food type had a different caloric density (Supplementary Table 1), total consumption was measured in summated $\mathrm{kCal}$ of each food type instead of total grams consumed.

\section{Cue-induced Suppression of Feeding Behavior}

Feeding procedures took place in sound attenuated operant chambers (Med Associates, Burlington, VT). The foods were placed in small foil receptacles. All foods were weighed before and after feeding sessions, which were carried out during the dark cycle. Rats were permitted $30 \mathrm{~min}$ access per day to the cafeteria diet (or chow in chow only rats) for 5 days to achieve stable food intake during the baseline period and then returned to home cages containing only chow. Baseline intake was recorded as the averaged kcal/ session over the final 3 days. Rats were then subjected to four conditioning sessions on consecutive days in the same operant chamber. During the $30 \mathrm{~min}$ conditioning sessions, a cue light was activated for $10 \mathrm{~min}$, turned off for $10 \mathrm{~min}$, and then turned back on for $10 \mathrm{~min}$. Punished rats received foot shock only during presentation of the cue light $(0.5 \mathrm{~mA}$ for $1.0 \mathrm{~s} ; 10$ stimulations with $\sim 1$ min intervals). No food was present in the chambers during these sessions. The day after the final conditioning session, rats were initially exposed to a 5 min light-paired reminder foot shock. Rats were removed from the chamber, then replaced and exposed to the cue light in the absence of foot shock for a 30 min session with the light activated intermittently (10 min on, $10 \mathrm{~min}$ off, $10 \mathrm{~min}$ on) in the presence of the cafeteria diet or chow for the chow only rats. In a separate experiment, rats were trained identically, except they were not exposed to the foot shock. Chow only animals received chow on the baseline and test days.

\section{Slice Preparation}

The day after the conditioned suppression test, rats were anesthetized with isoflurane decapitated, and coronal sections containing the lateral OFC were cut at $300 \mu \mathrm{m}$ on a vibratome (Leica, Nussloch, Germany) in solution containing (in $\mathrm{mM}$ ): 75 sucrose, $87 \mathrm{NaCl}, 2.5 \mathrm{KCl}, 1.25 \mathrm{NaH}_{2} \mathrm{PO} 4,25$ $\mathrm{NaHCO}_{3}, 7 \mathrm{MgCl}_{2}, 0.95 \mathrm{CaCl}_{2}$, and 3 kynurenic acid. Coronal sections were immediately transferred to $250 \mathrm{~mL}$ aCSF containing (in mM) $126 \mathrm{NaCl}, 1.6 \mathrm{KCl}, 1.1 \mathrm{NaH}_{2} \mathrm{PO}_{4}, 1.4$ $\mathrm{MgCl}_{2}, 26 \mathrm{NaHCO}_{3}, 11$ glucose, $2.4 \mathrm{CaCl}_{2}$, and incubated for a minimum of $60 \mathrm{~min}$ at $31.4-33^{\circ} \mathrm{C}$ prior to recording. All solutions were continuously saturated with $95 \% \mathrm{O}_{2} / 5 \% \mathrm{CO}_{2}$.

\section{Electrophysiology}

Slices were placed in the recording chamber and perfused with aCSF. Cells were visualized on an upright microscope using 'Dodt-type' gradient contrast optics (Dodt et al, 2002). Whole-cell voltage clamp recordings of lateral OFC pyramidal neurons in layer II/III were made using a Multiclamp 700B amplifier (Molecular devices, Union City, California). For voltage clamp experiments, recording electrodes (3-5 $\mathrm{M} \Omega$ ) were filled with (in $\mathrm{mM}$ ): 120 cesium methanesulfonate, 20 HEPES, 0.4 EGTA, 5 TEA-Cl, $2 \mathrm{MgCl}_{2}, 2.5$ MgATP, $0.5 \mathrm{NaGTP}$, and 5.4 biocytin (in some experiments), with a $\mathrm{pH}$ of $7.2-7.3$ and $280-295 \mathrm{mOsm}$. For current clamp experiments, recording electrodes were filled with (in $\mathrm{mM}$ ): 143 potassium-D-gluconate, $8 \mathrm{NaCl}, 2 \mathrm{MgCl}_{2}, 10$ HEPES, 0.2 EGTA, $2 \mathrm{Mg}$-ATP and $0.3 \mathrm{Na}_{2} \mathrm{GTP}$. To stimulate local presynaptic terminals, a tungsten bipolar stimulating electrode was placed $100-300 \mu \mathrm{m}$ following the apical dendrites of the neuron (distal to the recorded cell) and used to evoke paired pulses at $0.1 \mathrm{~Hz}$ with an inter-event interval of $50 \mathrm{~ms}$. Series resistance (6-20 M $\Omega$ ) and input resistance were monitored on-line with a $5-\mathrm{mV}$ depolarizing step $(50 \mathrm{~ms})$ given $300 \mathrm{~ms}$ prior to every afferent stimulus. Recordings exhibiting a $>20 \%$ change in series resistance were discarded. Excitatory postsynaptic currents (EPSCs) were recorded in the presence of picrotoxin $(50 \mu \mathrm{M})$ in order to block $\mathrm{GABA}_{\mathrm{A}}$ receptor-mediated inhibitory postsynaptic currents (IPSCs) while DNQX $(10 \mu \mathrm{M})$, strychnine $(1 \mu \mathrm{M})$, $\operatorname{DPCPX}(1 \mu \mathrm{M})$ and CGP-35348 $(1 \mu \mathrm{M})$ were added to isolate $\mathrm{GABA}_{\mathrm{A}}$ IPSCs. EPSCs and IPSCs were filtered at $2 \mathrm{kHz}$, digitized at $10 \mathrm{kHz}$ and collected on-line using pCLAMP 10 software. Currents traces were constructed by averaging 10 consecutive EPSC or IPSC sweeps.

AMPAR spontaneous EPSCs (sEPSCs) were recorded in cells voltage clamped at $-70 \mathrm{mV}$ in picrotoxin $(50 \mu \mathrm{M})$ and APV $(50 \mu \mathrm{M})$. Spontaneous IPSCs (sIPSCs) were recorded in cells voltage clamped at $+10 \mathrm{mV}$ in DNQX $(10 \mu \mathrm{M})$, Strychnine $(1 \mu \mathrm{M})$ and DPCPX $(1 \mu \mathrm{M})$, APV $(50 \mu \mathrm{M})$. Miniature EPSCs (mEPSCs) were recorded at $-70 \mathrm{mV}$ and miniature IPSCs (mIPSCs) were recorded at $+10 \mathrm{mV}$ in the 
presence of tetrodotoxin ( $500 \mathrm{nM}$ ) to block action potential driven spontaneous events. AMPAR mEPSCs were selected based on their amplitude ( $>12 \mathrm{pA})$, decay time $(<3 \mathrm{~ms})$, and rise time $(<1 \mathrm{~ms})$ using the MiniAnalysis program (Synaptosoft). Similarly, GABAA mIPSCs were selected for amplitude $(>12 \mathrm{pA})$, rise time $(<4 \mathrm{~ms})$, and decay time $(<10 \mathrm{~ms})$. Analysis of spontaneous and miniature events was performed blind to the experimental condition.

To investigate alterations in intrinsic excitability, neurons were held at $-75 \mathrm{mV}$ and depolarizing current steps $(1 \mathrm{~Hz}$, $300 \mathrm{~ms}$, from 0 to $+400 \mathrm{pA}$ in $50 \mathrm{pA}$ steps) were applied. These experiments were performed in the presence of excitatory and inhibitory synaptic blockers including DNQX $(10 \mu \mathrm{M})$, APV $(50 \mu \mathrm{M})$, strychnine $(1 \mu \mathrm{M})$, and picrotoxin $(100 \mu \mathrm{M})$.

\section{Golgi-Cox Impregnation}

In a separate cohort of rats fed a cafeteria diet or chow for 40 days, rats were deeply anesthetized with isoflurane, brains were rapidly extracted and blocks containing the OFC were immediately placed into Golgi-Cox fixative and processed using FD Rapid GolgiStain ${ }^{\mathrm{TM}}$ kit (FD Neurotechnologies). Impregnated blocks were cut in serial coronal $200 \mu \mathrm{m}$ thick sections using a vibratome. Sections were collected on gelatinized slides and mounted using a uniform orientation suitable for dendrite tracing and reconstruction. Sections were air-dried $(1 \mathrm{~h})$, incubated in the final staining solution, dehydrated in graded alcohols and mounted. Golgiimpregnated neurons, which were uniformly impregnated and not damaged by histological processing, were sampled under low power. Pyramidal neurons were identified by a pyramidal-like soma and a single prominent axon. Neurons were traced using a camera Lucida at $200 \mathrm{X}(21.17 \mu \mathrm{m} /$ concentric circle) for branching and 1000x $(10 \mu \mathrm{M}=1 \mathrm{~cm})$ for dendritic spines.

A Sholl analysis was used to characterize the morphology of Golgi-impregnated pyramidal neurons. The terminal tips from both the basilar and apical dendritic trees were identified. Spines were counted along consecutive $10 \mu \mathrm{m}$ fragments on secondary dendrites using an in-field frame always starting $10 \mu \mathrm{m}$ after branching from the primary dendrite. Only protrusions showing continuity with the dendritic shaft were considered for the analysis. All spine density and Sholl analyses were blinded to the animals' experimental condition.

\section{Statistics}

Bar graphs represent mean \pm s.e.m. $\mathrm{N}$ refers to either number of rats or number of neurons/rat. Statistical significance was assessed with a two-way ANOVA with a Dunnett's multiple comparison test using chow-fed rats as a control unless otherwise indicated. Statistics are presented in the figure legends.

\section{RESULTS}

Exposure to a cafeteria diet can induce obesity (Johnson and Kenny, 2010; Rolls et al, 1980). This is likely due to its high fat content and high glycemic load paired with the increased variety of accessible food (Rolls et al, 1983). To confirm that access to a cafeteria diet induces obesity, we measured daily weight gain over 40 days. Rats with extended access to a cafeteria diet gained significantly more weight than rats with restricted or no access to a cafeteria diet (Figure 1a). By the end of the diet exposure, rats with restricted or extended access to a cafeteria diet did not significantly differ in their consumption of fat, carbohydrate or protein (Figure 1b), suggesting that any behavioral or neuronal differences observed are not necessarily due to alterations in nutrient composition.

Rats with extended access to a cafeteria diet consumed significantly more calories over 40 days compared with chow-fed and restricted access rats (Figure 1c). The increased energy intake of extended access rats was mainly due to consumption of the cafeteria diet, even though they had access to ad libitum chow (Figure 1d). Averaged over the last 3 days of the diet, extended access rats consumed $8 \pm 0.6 \%$ of their diet from chow. Although restricted access rats maintained similar caloric intake as chow-fed rats, towards the end of the 40-day diet, restricted access rats obtained only $33 \pm 3 \%$ of their daily calories from chow (Figure 1d). Thus, these rats exhibit binge-like feeding behavior as, by day $40,67 \pm 3 \%$ of their daily caloric intake came from only $1 \mathrm{~h}$ access to the cafeteria diet. Consistent with obesogenic effects of a cafeteria diet, rats with extended access had increased plasma insulin and leptin compared with restricted access or chow-fed rats (Supplementary 1). Taken together, these data indicate that rats with extended access to a cafeteria diet gain more weight, consume more calories and have higher plasma insulin and leptin levels, consistent with obesity. Furthermore, rats with restricted access to a cafeteria diet binge-eat the majority of their daily calories during the $1 \mathrm{~h}$ access to the cafeteria diet even though they gain less weight than extended access rats.

A previous report demonstrated that rats with extended access to a cafeteria diet become resistant to punishment, such that they continue to eat in the presence of aversive cues (Johnson and Kenny, 2010). To test if rats with restricted or extended access to a cafeteria diet suppress feeding in the presence of aversive cues, we trained rats to consume the cafeteria diet in an operant chamber for $30 \mathrm{~min}$ for 5 days. Baseline energy intake was averaged over the last 3 days of intra-chamber feeding. Rats with restricted access to a cafeteria diet had elevated baseline feeding of cafeteria diet, consistent with a binge-like feeding pattern (Figure 1e). Baseline feeding was compared with consumption on the test day, whereby feeding was measured in the presence of a light previously paired with delivery of foot shocks. On the test day, rats with restricted access to a cafeteria diet suppressed their energy intake in the presence of aversive cues. In contrast, rats with extended access to a cafeteria diet did not suppress their feeding in the presence of the foot shockpredicting light (Figure 1e). In the absence of foot shocks paired to a light stimulus, neither restricted nor extended access rats altered their food intake compared with baseline (Figure 1f). Notably, chow-fed animals suppressed their feeding of chow in the presence of the foot shock-predicting light, but not in the absence of paired foot shocks, suggesting that even very low levels of standard chow consumed in the chambers can be further decreased in an aversive context. There was no correlation between the quantities eaten at baseline with the conditioned suppression in either group 
a

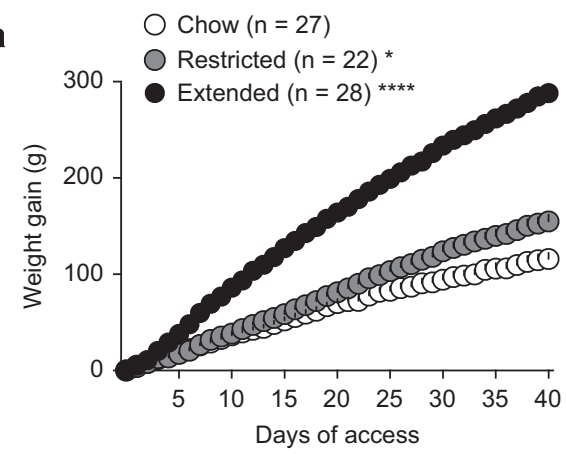

C
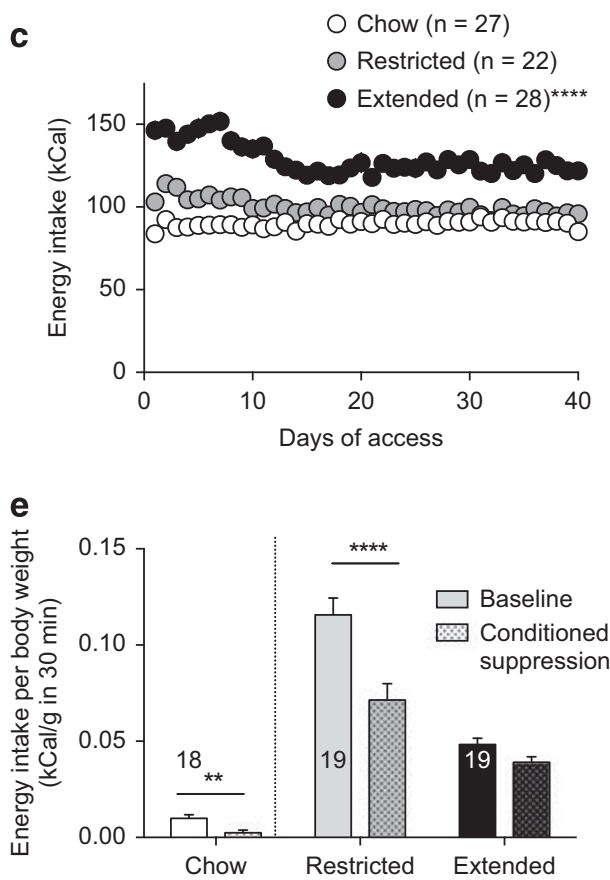

b

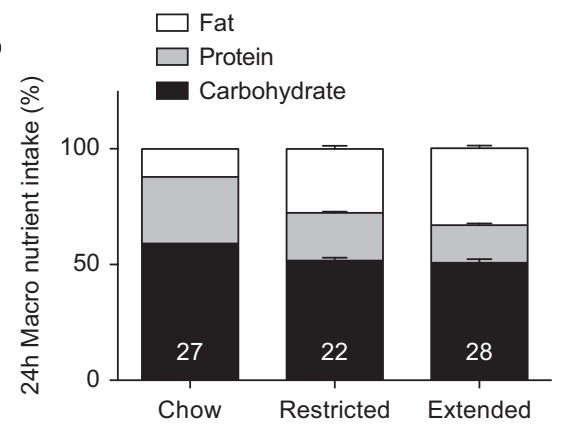

d
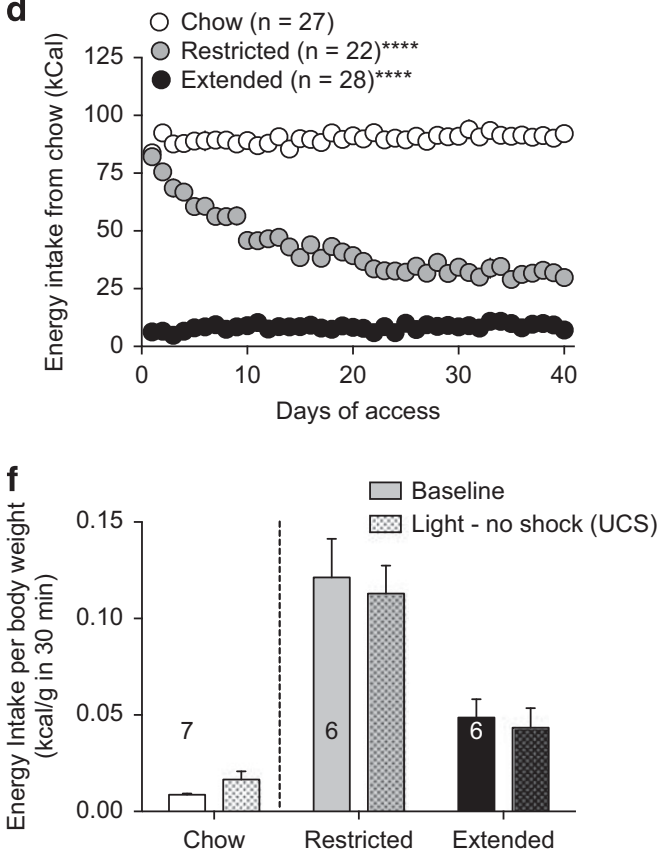

Figure I Rats with extended access to a cafeteria diet are obese and do not suppress feeding behavior in an aversive context. (a) Cumulative change in weight gain for extended $(23 \mathrm{~h}$ ) access (filled circles) restricted (I h) access (shaded circles) or chow-fed rats (open circles). There was a significant diet $\times$ time interaction on weight gain (RM two-way ANOVA, $(F(80,2920)=97.97, P<0.000 \mathrm{I})$. A Dunnett's multiple comparison test revealed a significant difference in weight gain between chow and extended $(P<0.000 \mathrm{I})$ or restricted $(P<0.05)$ access to a cafeteria diet. Weight gain on day 40 was significantly greater in extended access compared with restricted access or chow-fed rats (one-way ANOVA, F(2, 74) = 123.8). (b) Macronutrient consumption averaged over the final 3 days of the diet is not different between restricted and extended access groups (two-way ANOVA, interaction: $F(4,222)=\mid 08, P<0.001$; macronutrient: $F(2222)=1067, P<0.000$ I); diet: $F(2,222)=0.006, P=0.99)$. (c) Daily caloric intake from all foods in chow only, restricted access and extended access rats. There was a significant interaction of diet and time (RM two-Way ANOVA; Interaction: $F(78,2886)=3.658, P<0.000$ I, time: $F(39$, $2886)=6.38, P<0.000 \mathrm{I})$, diet: $F(2,74)=79.15, P<0.000 \mathrm{I})$. Rats with extended access to the cafeteria diet $(P<0.000 \mathrm{I})$ consume significantly more calories than rats with restricted access $(P<0.000 \mathrm{I})$ or chow-fed rats. (d) Daily chow consumed by chow-only, restricted access or extended access to a cafeteria diet. There was a significant interaction of diet and time (RM two-way ANOVA; Interaction of diet $\times$ time: $F(78,2886)=16.10, P<0.000$ I; diet: $F(2,74)=64.89$, $P<0.000$ I; time: $F(39,2886)=15.98, P<0.000 I)$. A Tukey's multiple comparison test revealed that extended $(P<0.000 \mathrm{I})$ and restricted access $(P<0.000 \mathrm{I})$ rats ate significantly more cafeteria diet than chow-fed rats. (e) Mean ( \pm s.e.m.) energy consumption per body weight from the cafeteria diet during a 30 min session prior to (baseline, filled bars) and after (test day, patterned bars) rats were conditioned that a light predicted an aversive foot shock. In the presence of foot-shock predicting cues, restricted access rats suppress feeding of the cafeteria diet, but extended access rats continue to consume the cafeteria diet regardless of aversive cues. (two-way RM ANOVA, Interaction: $F(I, 72)=7.44, P=0.008$, diet: $F(I, 72)=17.26, P<0.000 I$; suppression: $F(I, 72)=60.24$, $P<0.000$ I). A Bonferroni's multiple comparison test revealed a significant difference between baseline and suppressed consumption of restricted access rats $(P<0.000 \mathrm{I})$ but not extended access rats $(P>0.05)$. Ad libitium chow-fed rats significantly suppressed feeding of chow in the presence of aversive cues $(t(34)=3.17, P=0.0032)$. (f) When the house light was not paired with foot shocks there was no conditioned suppression of feeding in restricted access or extended access rats (2-way ANOVA, diet $\times$ suppression interaction, $F(I, I 0)=0.048, P=0.83$ ). Ad libitium chow-fed rats significantly suppressed feeding of chow in the presence of aversive cues $(\mathrm{t}(6)=1.8, P=0.12)$. Number on bars refers to $N($ rats). Bars or symbols represent mean \pm s.e.m. $* P<0.05, * * P<0.01$, ******* $<<0.0001$.

(extended access: $r^{2}=0.01, P=0.7, n=19$; restricted access: $\left.r^{2}=0.04, \quad P=0.4, \quad n=19\right)$, suggesting that the amount consumed in the baseline does not influence the ability to suppress feeding.
It has been proposed that obesity is associated with cognitive impairments (Beilharz et al, 2015; Winocur and Greenwood, 2005). To assess if rodents were able to learn to lever press for food, rats were trained to perform an 
instrumental response on a lever to receive a sucrose pellet. This response was paired with a cue light above the lever. The inactive lever had no consequence. There was no difference in the acquisition or expression of appetitive learning between rats with restricted or extended access to a cafeteria diet or chow-fed rats (Supplementary 2A,B). To test if access to a cafeteria diet influenced aversive learning, we measured freezing responses to the conditioned cue-light on the 4 th conditioning session in the $1 \mathrm{~min}$ prior to the rats receiving foot shocks or in separate animals where the light was presented without foot shocks. There were no significant differences in freezing between feeding groups amongst conditioned rats (Supplementary 2C), suggesting that the cafeteria diet does not influence aversive conditioning. To determine if extended access rats did not suppress their consumption during the aversive cue because they were unable to detect foot shocks, we assessed the number of startle responses during the last conditioning session. There were no significant differences between restricted, extended access or chow-fed rats (Supplementary 2D), indicating that obesity did not impair the ability to detect foot shocks. Taken together, rats with extended access to a cafeteria diet did not alter their food intake during aversive cues.

OFC function has been implicated in the devaluation of food rewards either by satiety (Gremel and Costa, 2013; Rudebeck et al, 2013), sickness paired cues (Schoenbaum et $\mathrm{al}, 2003$ ) or by changing the action contingency (Ostlund and Balleine, 2007). Therefore, we hypothesized that a cafeteria diet may induce changes in lateral OFC pyramidal neurons. To determine whether diet-induced obesity induced morphological changes to pyramidal neurons, we tested whether access to a cafeteria diet altered spine density on apical and basilar dendrites of Golgi-impregnated lateral, medial and ventral pyramidal neurons in layer II/III of the OFC. We found no significant effect of cafeteria diet on spine density in apical dendrites from any region tested (Figure 2a, $\mathrm{d}$ and $\mathrm{g}$ ). A two-way ANOVA revealed no main effects of diet treatment on apical spine density of any region $\left(\mathrm{F}_{(2}\right.$, $360)=2.299, P=0.1)$. In contrast, there was a significant main effect of diet treatment on basilar spine density $\left(\mathrm{F}_{(2}\right.$, 333) $=7.248 P=0.008)$. Extended access to a cafeteria diet decreased spine density in basal dendrites of layer II/III lateral OFC neurons (Figure 2b, c), but not in ventral (Figure $2 \mathrm{e}$ and $\mathrm{f}$ ) or medial OFC neurons (Figure 2h, i). Restricted access to a cafeteria diet increased spine density in basal dendrites of ventral OFC pyramidal neurons (Figure $2 \mathrm{e}$ and f), but not that of lateral or medial OFC neurons. We next tested if there were differences in dendritic branching of OFC pyramidal neurons in rats with extended, restricted or no access to a cafeteria diet. Branching of apical dendrites of lateral OFC neurons were not changed with access to a cafeteria diet (Figure 3a), but there was increased branching of basal dendrites with restricted or extended access to a cafeteria diet (Figure 3b). Taken together, access to a cafeteria diet alters the structural morphology of lateral OFC neurons.

Next, we tested if there were functional consequences of decreased basilar spine morphology and increased basal complexity of lateral OFC pyramidal neurons of obese rats. To determine if excitatory inputs to pyramidal neurons are altered by extended access to a cafeteria diet, we recorded sEPSCs (action potential dependent and independent) and mEPSCs (quantal) onto lateral OFC pyramidal neurons. Access to a cafeteria diet did not alter the amplitude or the frequency of sEPSCs, suggesting no change in glutamate release or its effects postsynaptically (Supplementary $3 \mathrm{~A}-\mathrm{C}$ ). Similarly, access to a cafeteria diet did not alter the amplitude or the frequency of mEPSCs onto pyramidal neurons (Supplementary 3D-E). To further assess if there were changes in glutamate release probability, we measured the paired pulse ratio with a $50 \mathrm{~ms}$ inter-stimulus interval, a measure that reliably correlates with probability of release (Thomson, 2000). However, there was no significant difference between diet groups (Supplementary 3F,G). Taken together, neither presynaptic glutamate release nor the number or function of postsynaptic AMPARs on pyramidal neurons were changed in rats with extended or restricted access to a cafeteria diet, suggesting that these effects are not a functional consequence of decreased spine density.

We next tested whether intrinsic properties of layer II/III lateral OFC pyramidal neurons were different between rats with restricted or extended access to a cafeteria diet and chow-fed rats. In the presence of excitatory and inhibitory synaptic blockers, there were no significant differences in current-step evoked firing between the feeding groups (Figure $4 \mathrm{a}$ and b). Furthermore, the spike latency of OFC pyramidal neurons from rats with extended access $(19 \pm 2 \mathrm{~ms})$ or restricted access $(19 \pm 3 \mathrm{~ms})$ to a cafeteria diet was not different from chow-fed rats ( $17 \pm 3 \mathrm{~ms}$; Figure $4 \mathrm{c})$. Taken together these data suggest that access to a cafeteria diet does not alter the intrinsic excitability of OFC pyramidal neurons.

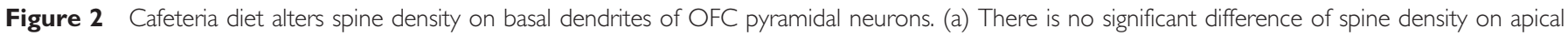

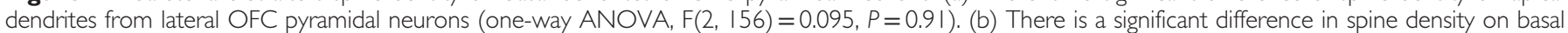

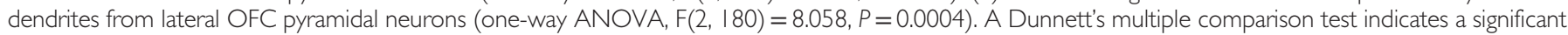





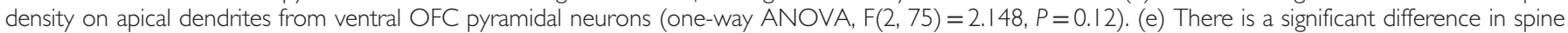

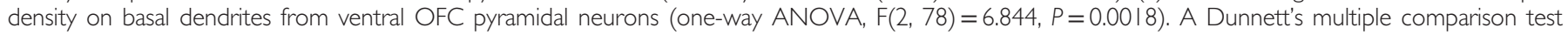

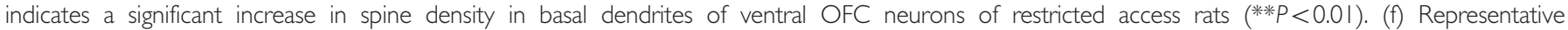



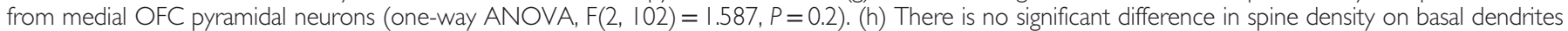

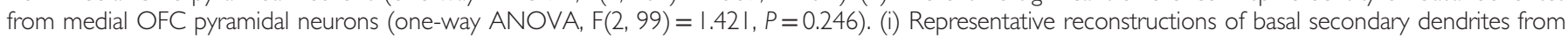



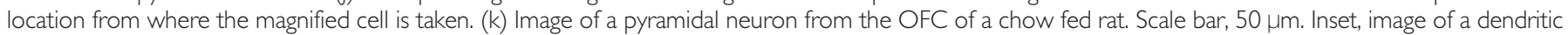

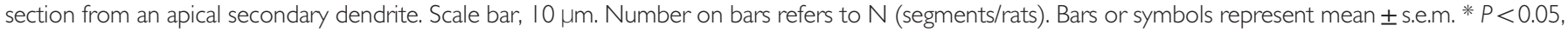
*** $P<0.01$
} 
We then measured the resting membrane potential in the presence or absence of synaptic blockers. In the absence of synaptic blockers, the resting membrane potential was significantly more depolarized in lateral OFC neurons of rats with extended access to a cafeteria diet compared with rats with restricted access or chow-fed rats (Figure $4 \mathrm{~d}$ ). However, this effect was absent in the presence of synaptic blockers (Figure 4e). Taken together, these data suggest that lateral OFC neurons from rats with extended access to a cafeteria diet are more depolarized due to an alteration in synaptic input, but not a change in intrinsic excitability.

We next tested whether the depolarized resting membrane potential was due to a suppression of inhibitory synaptic input onto lateral OFC pyramidal neurons. OFC pyramidal
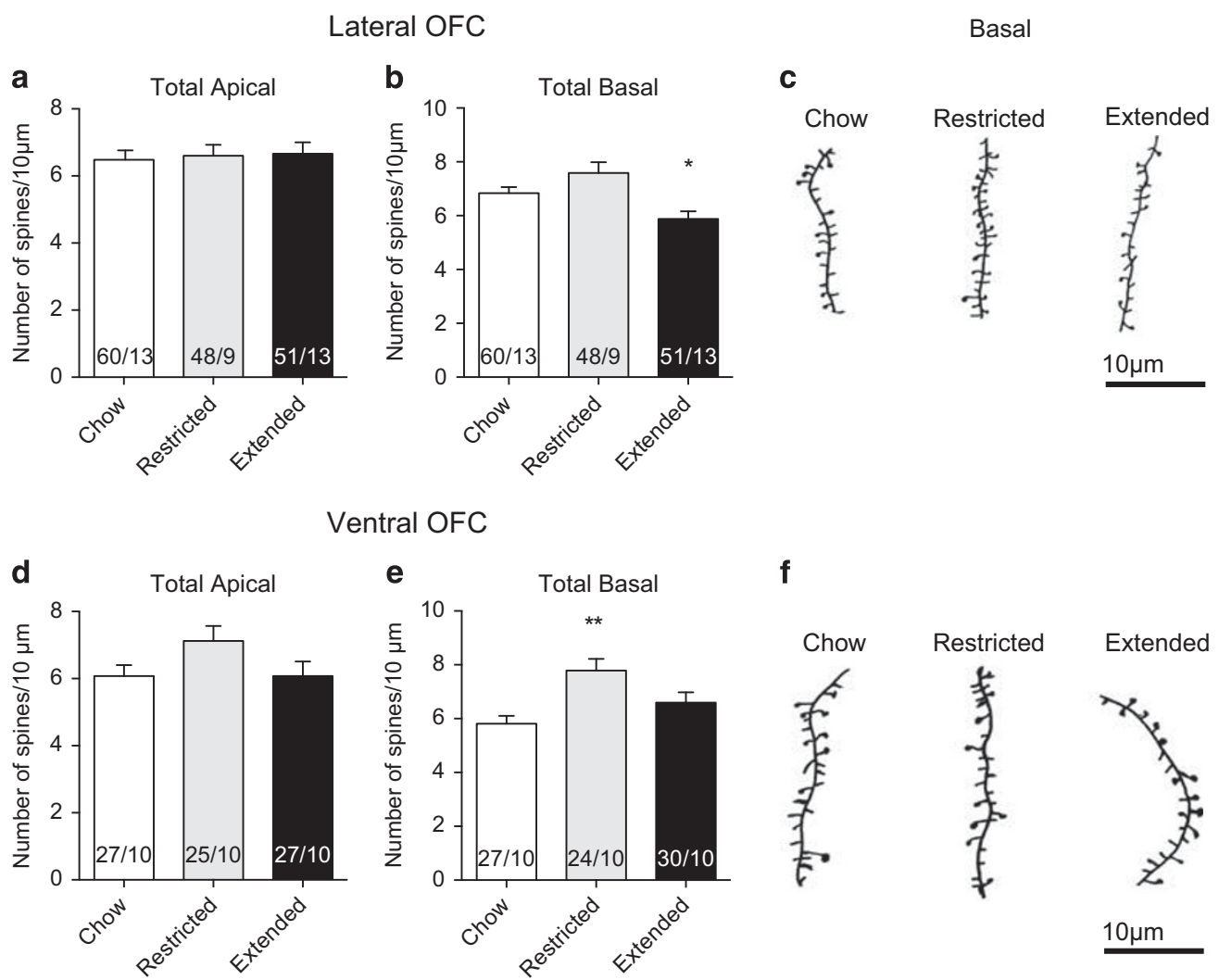

f
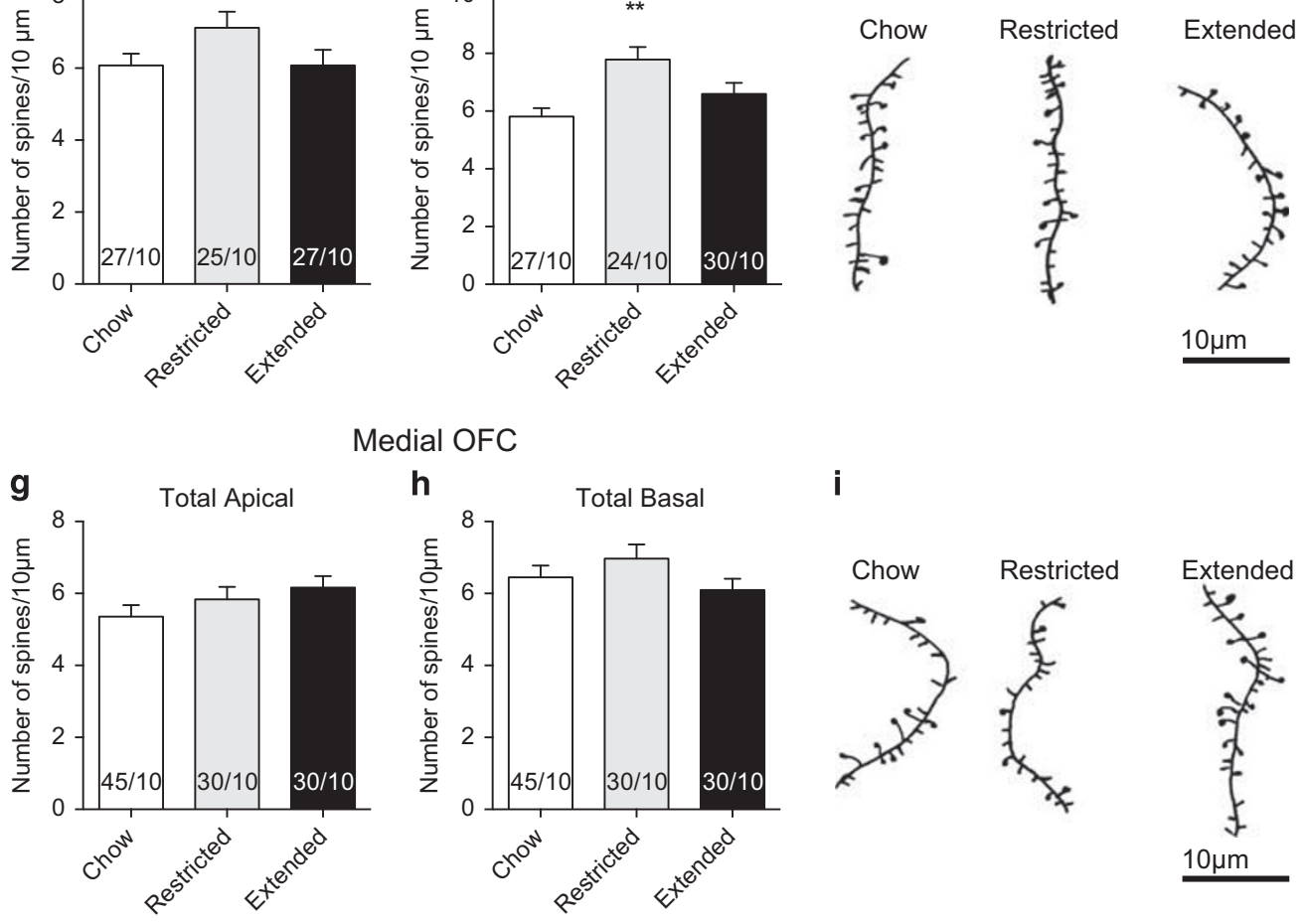

Medial OFC

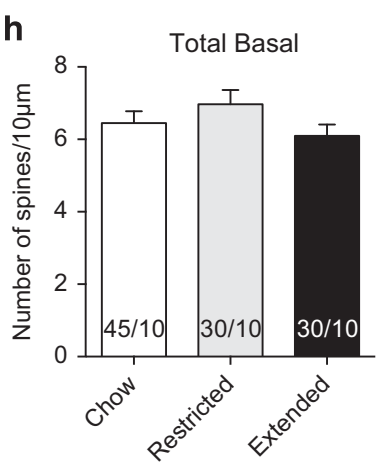

i




Figure 3 Cafeteria diet alters basal dendritic morphology of lateral OFC neurons. (a) There are no significant differences in dendritic branching of apical dendrites of lateral OFC neurons between feeding groups (two-way RM ANOVA: diet $\times$ radius interaction, $F(42,1680)=1.086, P=0.327$ ). (b) There is a significant interaction of diet $\times$ radius on dendritic branching of basal dendrites of lateral OFC neurons (two-way RM ANOVA: diet $\times$ radius interaction, $F(20,820)=3.175, P<0.0001)$. A Dunnett's multiple comparison test reveals a significant increase of basal dendritic branching of lateral OFC neurons from restricted access $(P<0.0 \mathrm{I})$ or extended access $(P<0.05)$ rats compared with chow-fed rats. (c) Representative camera lucida drawing of a lateral OFC pyramidal neuron from a chow-fed rat. Overlaid are a number of concentric rings with regular radial increments centered in the neuronal soma for the Sholl analysis. The number of neurites intersecting each ring of increasing radii is counted and averaged over a population of sampled neurons. $\mathrm{N}$ refers to cell/rats. Symbols represent mean \pm s.e.m. $* P<0.05$, $* * P<0.01$.

neurons receive a variety of local inhibitory inputs from GABAergic interneurons that make perisomatic synapses on multiple pyramidal neurons to control their firing and synchronicity (Quirk et al, 2009). Therefore, it is possible that extended access to a cafeteria diet may reduce inhibitory inputs onto lateral OFC pyramidal neurons, thus depolarizing the resting membrane potential. We tested this hypothesis by measuring sIPSCs and mIPSCs. The frequency of sIPSCs onto lateral OFC neurons from extended access rats was significantly decreased compared with chow-fed rats (Figure $5 \mathrm{a}$ and $\mathrm{c}$ ), suggesting a change in the presynaptic release probability of GABA at inhibitory synapses onto lateral OFC pyramidal neurons. Correspondingly, we observed a decrease in the frequency of mIPSCs compared with chow-fed rats (Figure 5e). There were no significant differences in sIPSC or mIPSC amplitude between extended access, restricted access or chow-fed rats, suggesting no alterations in postsynaptic number or function of $\mathrm{GABA}_{\mathrm{A}}$ receptors (Figure 5a,b and d). To confirm a change in GABA release probability, we measured the ratio of evoked paired pulses of $\mathrm{GABA}_{\mathrm{A}}$ receptor IPSCs. The paired-pulse ratio of lateral OFC pyramidal neurons from extended rats was significantly increased compared with restricted access or chow-fed rats (Figure $5 \mathrm{f}$ and g). Taken together, these data indicate that inhibitory inputs to lateral OFC pyramidal neurons are diminished in rats with extended access to a cafeteria diet.

An alternate mechanism for decreased inhibitory synaptic input to lateral OFC pyramidal neurons could be due to decreased expression of GABAergic interneurons. Cortical inhibitory interneurons expressing parvalbumin $(\mathrm{PV}+)$ are regulators of local network activities such that activation of $\mathrm{PV}+$ neurons can synchronize networks of pyramidal neurons into gamma oscillations (Buzsáki and Wang, 2012). Decreased cortical PV+ interneurons have been implicated in a number of mental illnesses that dysregulate prefrontal information processing (Coyle, 2004; Del Pino et al, 2013; Lewis et al, 2012; Volman et al, 2011). Therefore, we assessed if access to a cafeteria diet altered the number of $\mathrm{PV}+$ interneurons in the lateral OFC. However, there was no 
b

Chow

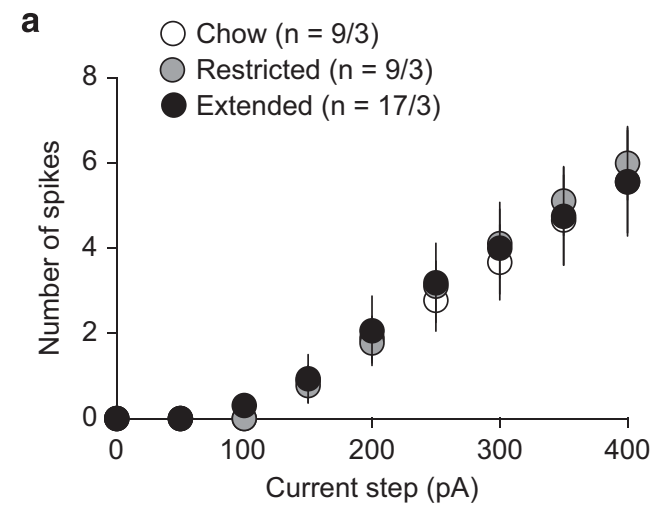

Restricted



C

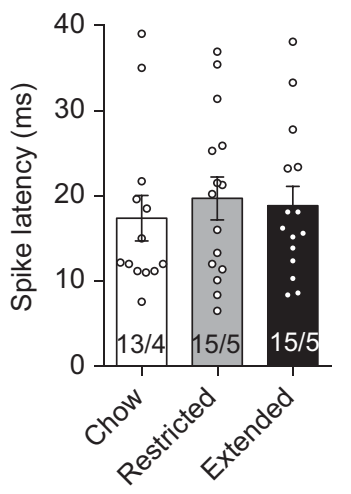

d

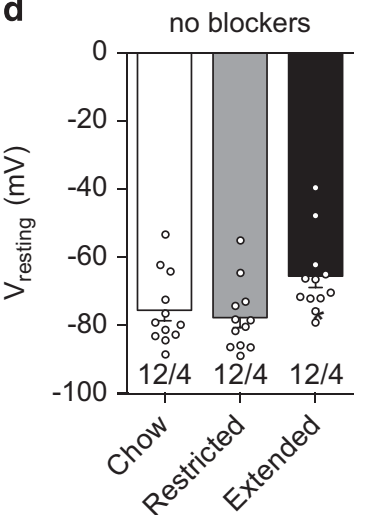

Figure 4 Extended access to a cafeteria diet does not alter intrinsic excitability of lateral OFC pyramidal neurons. (a) Current steps from 0 to 400 pA were used to evoke firing in the presence of synaptic blockers, including presence of AMPA (DNQX, I HM), NMDA (AP-5, $50 \mu M)$, Glycine (Strychnine, I0 HM), or $\mathrm{GABA}_{\mathrm{A}}$ (picrotoxin, $100 \mu \mathrm{M}$ ) receptor blockers. There was no significant difference in intrinsic excitability of lateral OFC pyramidal neurons from rats with extended access to a cafeteria diet (ANOVA $F(2,48)=0.018, P=0.98$ ). (b) Example recordings of evoked firing from a 350 pA current step from lateral OFC pyramidal neurons of chow only, restricted access or extended access rats. (c) Latency to the first spike of lateral OFC pyramidal neurons in the presence of synaptic blockers is not significantly different between feeding groups (one-way ANOVA, $F(2,40)=0.21, P=0.81$ ). (d) Resting membrane potential was recorded immediately after obtaining whole cell configuration in current-clamp mode. Pyramidal neurons from extended access rats were significantly more depolarized than those from restricted access or chow fed rat (one-way ANOVA, $F(2,33)=4.387, P=0.02$ ). (e) In the presence of synaptic blockers, resting membrane potential of lateral OFC pyramidal neurons is not significantly different (one-way ANOVA, $F(2,40)=1.582, P=0.22$ ). Number on graphs refers to $N$ (cells/rats). Symbols on bars represent individual cells. Bars or symbols represent mean \pm s.e.m. $* P<0.05$.

significant difference in the number of PV+ neurons in the lateral OFC of rats with extended, restricted or no access to a cafeteria diet (Supplementary 4A,B). Taken together, while extended access to a cafeteria diet reduced GABA release onto pyramidal neurons, this was not due to a change in number of $\mathrm{PV}+$ interneurons.

\section{DISCUSSION}

Hyperphagia, driven by the availability of palatable food with a high-fat content and high glycemic load, is the largest determinant of obesity (Gearhardt et al, 2011; Kelly et al, 2009; Popkin and Duffey, 2010). Individuals often overeat regardless of known aversive consequences. Dysfunction of the OFC has been implicated in maladaptive decision making (Wilson et al, 2014). Here, we demonstrate that obese rats have altered basilar spine density and dendritic branching as well as reduced probability of GABA release onto layer II/III lateral OFC pyramidal neurons. These animals also exhibited impairments in flexible behavior such that they did not suppress feeding in the presence of aversive cues. These results point to a potential mechanism underlying non-homeostatic feeding.

\section{Obese Rats do not Inhibit Food Consumption in the Presence of Aversive Cues}

Consistent with previous work (Johnson and Kenny, 2010), rats with extended access to a cafeteria diet continue to eat regardless of aversive cues. We propose that this is due to a change in response priority due to the incentive value of the food and not a change in Pavlovian or instrumental learning, as obese rats were capable of both appetitive and aversive conditioning. Consistent with these findings, others have demonstrated that cafeteria-diet-induced obesity show increased conditioned approach behaviors likely to due to the enhanced incentive value of the food without a change in the rate of acquisition of Pavlovian learning (Robinson et al, 
a


Figure 5 Rats with extended access to a cafeteria diet have decreased GABA release onto pyramidal neurons. (a) Example traces of spontaneous inhibitory post-synaptic currents (sIPSC; recorded at $+10 \mathrm{mV}$ ) from lateral OFC pyramidal neurons from chow-fed, restricted or extended access rats. (b) Amplitude of sIPSCs onto lateral OFC pyramidal neurons was not significantly different between feeding groups (one-way ANOVA, $F(2,63)=1.68, P=0.19$ ). (c) sIPSC frequency was significantly less in lateral OFC pyramidal neurons from rats with extended access to a cafeteria diet (one-way ANOVA, F $(2,65)=3.74$, $P=0.033)$. Dunnett's multiple comparison test revealed a significant difference between extended access compared with chow-fed controls, $* P<0.05$. (d) mIPSC amplitude was not significantly different between feeding groups (one-way ANOVA, $F(2,60)=0.09, P=0.9 \mathrm{I}$ ). (e) Frequency of mIPSC events onto lateral OFC pyramidal neurons were significantly decreased in rats with extended access to a cafeteria diet (one-way $A N O V A, F(2,61)=3.44, P=0.03)$. $A$ Dunnett's multiple comparison test revealed a significant difference between extended access compared with chow-fed controls, * $P<0.05$. $(f)$ Mean $( \pm$ s.e.m.) of paired pulse ratio $(50 \mathrm{~ms}$ interval) of lateral OFC neurons indicates a significant paired pulse facilitation suggesting decreased release probability at GABAergic terminals onto pyramidal neurons of extended access rats (one-way ANOVA, $F(2,44)=5.54, P=0.007$ ). Dunnett's multiple comparison test revealed a significant difference between extended access compared with chow-fed controls, $* * P<0.01$. (g) Example traces of paired pulses evoked at $+10 \mathrm{mV}$ at inhibitory synapses onto lateral OFC pyramidal neurons from chow-fed, restricted and extended access rats. Number below or on bars refers to $\mathrm{N}$ (cells/rats). Symbols represent individual cells. Bars represent mean \pm s.e.m.

2015). Rats with restricted access to a cafeteria diet exhibited binge-like eating behavior such that they consumed $2 / 3$ of their daily caloric needs within $1 \mathrm{~h}$ and that they had elevated baseline feeding in the operant chambers compared with extended access rats. Even though the restricted access rats binge-feed and derive a similar proportion of macronutrient content from the cafeteria diet as the extended access rats, they inhibited their feeding in the presence of aversive cues.
This suggests that the inability to change feeding behavior under threat may be associated with obesity rather than the nutritional components of the diet per se. An increase in food consumption by extended access rats during the aversive cue is not likely due to stress-induced feeding as restricted access rats suppress their feeding and typically stress-induced feeding responses occur after the animal has been removed from the stressor (Maniam and Morris, 2012). 
Rats with Extended Access to a Cafeteria Diet have Decreased Probability of Release of GABA onto Pyramidal Neurons of The Lateral OFC

Previous work has demonstrated that decreased striatal dopamine D2 receptor expression underlies continued feeding during aversive cues (Johnson and Kenny, 2010). We found that obese rats had both a decreased inhibitory input to OFC pyramidal neurons as well as impairment in the ability to appropriately organize their response priority. Presumably, decreased inhibitory input would disinhibit pyramidal neurons. Indeed, lateral OFC pyramidal neurons from extended access rats had a depolarized resting membrane potential. Consistent with our results, optogenetic stimulation of CaMKII-containing OFC neurons leads to increased lever pressing for sucrose in the satiety-induced devalued state (Gremel and Costa, 2013). Notably, these electrophysiological changes were not observed in rats with restricted access to the cafeteria diet that suppressed their feeding in the aversive context. This decrease in GABA release probability was evident by decreased sIPSC and mIPSC frequency and an increase in the paired pulse ratio. Distributed amongst pyramidal neurons in the OFC are fastspiking GABAergic interneurons, which generate narrow action potentials and are often PV+ or cholecystokinincontaining (Kawaguchi and Kubota, 1997). Given their cellular and anatomical properties, fast-spiking neurons are well positioned to coordinate the spiking activity of OFC pyramidal cells (Quirk et al, 2009). Previous work has demonstrated that Plaur mice with reduced PV+ interneurons exhibited impaired cognitive flexibility on a spatial reversal learning task with altered neuronal firing in the OFC (Bissonette et al, 2014). We did not observe differences in number of PV+ neurons in the OFC of rats exposed to a cafeteria diet or chow. Because diet-induced obesity increases 2-arachidonoylglycerol and anandamide in the hippocampus (Massa et al, 2010), an intriguing possibility is that extended access to a cafeteria diet increases endocannabinoidmediated suppression of inhibitory inputs to OFC pyramidal neurons. Future experiments should be aimed at addressing this possibility.

\section{Rats with Extended Access to a Cafeteria Diet have Altered Structural Plasticity}

Decreased GABAergic input to pyramidal neurons is unlikely to be reflected in changed spine density as most GABAergic inputs synapse perisomatically or axo-axonally onto inputs to cortical dendrites (Somogyi et al, 1998). Extended access to a cafeteria diet did not alter spine density at apical dendrites, but decreased spine density at basilar dendrites of lateral OFC neurons. Similarly, obese rats or animals on a high fat diet had decreased spine density in other areas of the prefrontal cortex (Bocarsly et al, 2015; Dingess et al, 2016). Surprisingly, we did not observe an alteration in excitatory inputs to pyramidal neurons that would presumably be a functional representation of decreased spine density. However, the apical tuft of pyramidal neurons receives excitatory synaptic inputs that have different presynaptic origins to those that form synapses onto basal dendrites, which purportedly receive inputs from local excitatory input (Spruston, 2008). Thus, our whole cell recordings may reflect a different population of excitatory synapses on spines than that assessed with Golgi staining. Although there was a decrease in spine density on basal dendrites of lateral OFC neurons from obese rats, we observed an increase in basilar, but not apical, dendritic complexity in rats with extended or restricted access to a cafeteria diet compared with controls. The distinct morphologies of basal and apical dendrites suggest that inputs to these domains might be integrated differently. However, it is unknown what the functional implications may be of enhanced basal dendritic complexity of lateral OFC neurons.

Although restricted access rats did not show alterations in excitatory or inhibitory synaptic transmission, there was a significant increase in spine density in the ventral OFC. The ventral OFC is thought to bridge the medial and prefrontal cortical networks (Price, 2007), with the dorsal striatum being their primary extracortical target (Hoover and Vertes, 2011). While restricted access rats serve as a non-obese control with exposure to the diet in these studies, they do exhibit disordered feeding behavior such that they consume most of their daily energy requirement within an hour. Thus, future studies could test whether the ventral OFC projection to the striatum is involved in habit-like responding associated with binge eating.

In conclusion, we provide a novel hypothesis for how obesity may influence non-homeostatic eating. We demonstrate that obese rats, which do not suppress feeding in the presence of aversive cues, have decreased probability of GABA release onto OFC pyramidal neurons. This effect may contribute to increased excitability of lateral OFC neurons due to a more depolarized resting state. Thus, output from layer II/III OFC neurons may lack appropriate synchronicity to convey information to their target neurons. Because the OFC integrates sensory information with current reward value to guide future actions, it is likely that obesity-induced dysfunction in the OFC may lead individuals to eat regardless of homeostatic needs, further contributing to overeating.

\section{FUNDING AND DISCLOSURE}

This work was supported by a CIHR FDN: 148473, a Mind Foundation of British Columbia Young Investigator Award, and a Cumming School of Medicine Bridge fund to SLB. The authors declare no conflict of interest.

\section{REFERENCES}

Beilharz JE, Maniam J, Morris MJ (2015). Diet-induced cognitive deficits: the role of fat and sugar, potential mechanisms and nutritional interventions. Nutrients 7: 6719-6738.

Bissonette GB, Schoenbaum G, Roesch MR, Powell EM (2015). Interneurons are necessary for coordinated activity during reversal learning in orbitofrontal cortex. Biol Psychiatry 77: 454-464.

Bocarsly ME, Fasolino M, Kane GA, LaMarca EA, Kirschen GW, Karatsoreos IN et al (2015). Obesity diminishes synaptic markers, alters microglial morphology, and impairs cognitive function. Proc Natl Acad Sci USA 112: 15731-15736.

Berridge KC (1991). Modulation of taste affect by hunger, caloric satiety, and sensory-specific satiety in the rat. Appetite 16: 103-120. 
Buzsáki G, Wang X-J (2012). Mechanisms of gamma oscillations. Annu Rev Neurosci 35: 203-225.

Coyle JT (2004). The GABA-glutamate connection in schizophrenia: which is the proximate cause? Biochem Pharmacol 68: 1507-1514

Del Pino I, García-Frigola C, Dehorter N, Brotons-Mas JR, AlvarezSalvado E, Martínez de Lagrán M et al (2013). Erbb4 deletion from fast-spiking interneurons causes schizophrenia-like phenotypes. Neuron 79: 1152-1168.

Dingess PM, Darling RA, Kurt Dolence E, Culver BW, Brown TE (2016). Exposure to a diet high in fat attenuates dendritic spine density in the medial prefrontal cortex. Brain Struct Funct. (doi:10.1007/s00429-016-1208-y; e-pub ahead of print).

Dodt HU, Eder M, Schierloh A, Zieglgansberger W (2002). Infrared-guided laser stimulation of neurons in brain slices. Sci STKE 2002: pl2.

Finucane MM, Stevens GA, Cowan MJ, Danaei G, Lin JK, Paciorek CJ et al (2011). National, regional, and global trends in body-mass index since 1980: systematic analysis of health examination surveys and epidemiological studies with 960 country-years and 9.1 million participants. Lancet Lond Engl 377: 557-567.

Gearhardt AN, Davis C, Kuschner R, Brownell KD (2011). The addiction potential of hyperpalatable foods. Curr Drug Abuse Rev 4: 140-145.

Gremel CM, Costa RM (2013). Orbitofrontal and striatal circuits dynamically encode the shift between goal-directed and habitual actions. Nat Commun 4: 2264.

Hoover WB, Vertes RP (2011). Projections of the medial orbital and ventral orbital cortex in the rat. J Comp Neurol 519: 3766-3801.

Horstmann A, Dietrich A, Mathar D, Pössel M, Villringer A, Neumann J (2015). Slave to habit? Obesity is associated with decreased behavioural sensitivity to reward devaluation. Appetite 87: 175-183.

Johnson PM, Kenny PJ (2010). Dopamine D2 receptors in addiction-like reward dysfunction and compulsive eating in obese rats. Nat Neurosci 13: 635-641.

Kawaguchi Y, Kubota Y (1997). GABAergic cell subtypes and their synaptic connections in rat frontal cortex. Cereb Cortex 7: 476-486.

Kelly MT, Rennie KL, Wallace JMW, Robson PJ, Welch RW, Hannon-Fletcher MP et al (2009). Associations between the portion sizes of food groups consumed and measures of adiposity in the British National Diet and Nutrition Survey. Br J Nutr 101: 1413-1420.

Lewis DA, Curley AA, Glausier JR, Volk DW (2012). Cortical parvalbumin interneurons and cognitive dysfunction in schizophrenia. Trends Neurosci 35: 57-67.

Maniam J, Morris MJ (2012). The link between stress and feeding behaviour. Neuropharmacology 63: 97-110.

Massa F, Mancini G, Schmidt H, Steindel F, Mackie K, Angioni C et al (2010). Alterations in the hippocampal endocannabinoid system in diet-induced obese mice. J Neurosci Off J Soc Neurosci 30: 6273-6281.

Ostlund SB, Balleine BW (2007). Orbitofrontal cortex mediates outcome encoding in Pavlovian but not instrumental conditioning. J Neurosci Off J Soc Neurosci 27: 4819-4825.

Popkin BM, Duffey KJ (2010). Does hunger and satiety drive eating anymore? Increasing eating occasions and decreasing time between eating occasions in the United States1234. Am J Clin Nutr 91: 1342-1347.

Price JL (2007). Definition of the orbital cortex in relation to specific connections with limbic and visceral structures and other cortical regions. Ann NY Acad Sci 1121: 54-71.

Quirk MC, Sosulski DL, Feierstein CE, Uchida N, Mainen ZF (2009). A defined network of fast-spiking interneurons in orbitofrontal cortex: responses to behavioral contingencies and ketamine administration. Front Syst Neurosci 3: 13.

Reichelt AC, Morris MJ, Westbrook RF (2014). Cafeteria diet impairs expression of sensory-specific satiety and stimulusoutcome learning. Front Psychol 5: 852.

Robinson MJF, Burghardt PR, Patterson CM, Nobile CW, Akil H, Watson SJ et al (2015). Individual differences in cue-induced motivation and striatal systems in rats susceptible to diet-induced obesity. Neuropsychopharmacol Off Publ Am Coll Neuropsychopharmacol 40: 2113-2123.

Rolls BJ, Hetherington M, Burley VJ (1988). The specificity of satiety: the influence of foods of different macronutrient content on the development of satiety. Physiol Behav 43: 145-153.

Rolls BJ, Rowe EA, Turner RC (1980). Persistent obesity in rats following a period of consumption of a mixed, high energy diet. J Physiol 298: 415-427.

Rolls BJ, Van Duijvenvoorde PM, Rowe EA (1983). Variety in the diet enhances intake in a meal and contributes to the development of obesity in the rat. Physiol Behav 31: 21-27.

Rolls ET (2012). Taste, olfactory and food texture reward processing in the brain and the control of appetite. Proc Nutr Soc 71: 488-501.

Rudebeck PH, Saunders RC, Prescott AT, Chau LS, Murray EA (2013). Prefrontal mechanisms of behavioral flexibility, emotion regulation and value updating. Nat Neurosci 16: 1140-1145.

Schoenbaum G, Setlow B, Saddoris MP, Gallagher M (2003). Encoding predicted outcome and acquired value in orbitofrontal cortex during cue sampling depends upon input from basolateral amygdala. Neuron 39: 855-867.

Somogyi P, Tamás G, Lujan R, Buhl EH (1998). Salient features of synaptic organisation in the cerebral cortex. Brain Res Brain Res Rev 26: 113-135.

Spruston N (2008). Pyramidal neurons: dendritic structure and synaptic integration. Nat Rev Neurosci 9: 206-221.

Stoeckel LE, Cox JE, Cook EW, Weller RE (2007). Motivational state modulates the hedonic value of food images differently in men and women. Appetite 48: 139-144.

Swinburn B, Sacks G, Ravussin E (2009). Increased food energy supply is more than sufficient to explain the US epidemic of obesity. Am J Clin Nutr 90: 1453-1456.

Thomson AM (2000). Facilitation, augmentation and potentiation at central synapses. Trends Neurosci 23: 305-312.

Volman V, Behrens MM, Sejnowski TJ (2011). Downregulation of parvalbumin at cortical GABA synapses reduces network gamma oscillatory activity. J Neurosci Off J Soc Neurosci 31: 18137-18148.

Wilson RC, Takahashi YK, Schoenbaum G, Niv Y (2014). Orbitofrontal cortex as a cognitive map of task space. Neuron 81: 267-279.

Winocur G, Greenwood CE (2005). Studies of the effects of high fat diets on cognitive function in a rat model. Neurobiol Aging 26 (Suppl 1): 46-49.

Supplementary Information accompanies the paper on the Neuropsychopharmacology website (http://www.nature.com/npp) 